\title{
Ergodic averages with generalized weights
}

\author{
by \\ DoĞAn Çömez (Fargo, ND) and Semyon N. Litvinov (Hazleton, PA)
}

\begin{abstract}
Two types of weighted ergodic averages are studied. It is shown that if $F=\left\{F_{n}\right\}$ is an admissible superadditive process relative to a measure preserving transformation, then a Wiener-Wintner type result holds for $F$. Using this result new good classes of weights generated by such processes are obtained. We also introduce another class of weights via the group of unitary functions, and study the convergence of the corresponding weighted averages. The limits of such weighted averages are also identified.
\end{abstract}

1. Introduction. This article is inspired by the celebrated WienerWintner theorem which has been instrumental in studying various properties of weighted and subsequential averages as well as constructing some nontrivial classes of good sequences. It turns out that, utilizing the tools developed in $[\mathrm{C}, \mathrm{CF}]$, one can obtain a version of this theorem in the setting of a class of superadditive processes. In turn, this superadditive version of Wiener-Wintner theorem leads to new classes of weights. In Sections 2-4 of this article, besides proving these results, we will also study convergence of sequences of weights defined by such superadditive processes.

The study of the behavior of the ergodic averages modulated by means of Besicovitch's sequences was initiated by C. Ryll-Nardzewski [Ry] (for $p=1$ ), and A. Tempelman [T] (for $p>1$ and in the context of Besicovitch functions on LCA groups). Later, various generalizations of this result were obtained [BeL, JO, LO, LOT]. Recently, another such attempt was made in [Li] in the non-commutative setting. The tools utilized in [Li] have some interesting ramifications in the commutative case as well. In Sections 5 and 6 of this article, following an observation made on the Wiener-Wintner theorem and adapting some of the techniques of [Li], we arrive at two rather distinct weighted ergodic theorems in the spirit of [Ry]. First we define a generalized Besicovitch sequence associated with a subset of the group of unimodular

2000 Mathematics Subject Classification: Primary 28D05; Secondary 37A99.

Key words and phrases: weighted and subsequential ergodic theorems, admissible processes, bounded Besicovitch sequences.

Research of D. Çömez supported in part by ND-EPSCoR through NSF OSR-9452892. 
functions in $L_{\infty}$, and then study convergence of the corresponding weighted averages. In the case of numerical Besicovitch sequences, which are discussed in $[R y]$, the role of such a subset is played by the unit circle in $\mathbb{C}$. This type of weights (generated by the unimodular group) is fundamentally different from the ones studied in [LO]. Section 7 is devoted to the identification of the limits of the weighted averages along generalized Besicovitch sequences.

Let $(X, \Sigma, \mu)$ be a probability space, and $T: X \rightarrow X$ be an invertible measure preserving transformation (MPT). Often, we will write $T^{i} f$ instead of $f \circ T^{i}$. A sequence $\mathbf{n}=\left\{n_{k}\right\}$ of positive integers (or a weight $\mathbf{a}=\left\{a_{i}\right\}$ ) is called good in the $p$-mean for $T$ if the limit of the corresponding averages $N^{-1} \sum_{i=0}^{N-1} f\left(T^{n_{i}} x\right)$ (or $\left.N^{-1} \sum_{i=0}^{N-1} a_{i} T^{i} f(x)\right)$ exists in $L_{p}$-norm for all $f \in$ $L_{p}$, and is called good a.e. in $L_{p}$ for $T$ if the limit of the averages exists a.e. for all $f \in L_{p}$. If $\mathbf{n}=\left\{n_{k}\right\}$ (or $\mathbf{a}=\left\{a_{i}\right\}$ ) is good a.e. (good in the $p$-mean) in $L_{p}$ for all MPTs, it is called good a.e. in $L_{p}$ (good in the p-mean).

A family $F=\left\{f_{n}\right\}_{n \geq 0} \subset L_{p}$ is called a $T$-superadditive process if the sequence $\left\{F_{n}\right\}$ of partial sums, where $F_{0}=0$ and $F_{n}=\sum_{i=0}^{n-1} f_{i}$, satisfies $F_{n+m} \geq F_{n}+T^{n} F_{m}$ for all $n, m \geq 0$. If the equality holds, it is called T-additive, and if the reverse inequality holds, it is called T-subadditive. $T$-additive processes are necessarily of the form $F_{n}=\sum_{i=0}^{n-1} T^{i} f_{0}$. If $\sup _{n \geq 1} n^{-1}\left\|F_{n}\right\|_{p}<\infty$, then the process $F \subset L_{p}$ is called $p$-bounded (or simply bounded when $p=1$ ). For a $T$-superadditive process $F, F_{n} \geq \sum_{i=0}^{n-1} T^{i} f_{0}$ for all $n \geq 1$, hence $F_{n}^{\prime}=F_{n}-\sum_{i=0}^{n-1} T^{i} f_{0}$ is a positive superadditive process (i.e. $F_{n}^{\prime} \geq 0$ for all $\mathrm{n}$ ) and is necessarily increasing. It follows that, if a result is valid for additive processes, then the same holds for $F$ if and only if it holds for $F^{\prime}$.

Given a sequence $\mathbf{n}=\left\{n_{k}\right\}$ or a weight $\mathbf{a}=\left\{a_{i}\right\}$ and a $T$-superadditive process $F$, we will define corresponding subsequential averages as $N^{-1} \sum_{i=0}^{N-1} f_{n_{i}}$ and the weighted averages as $N^{-1} \sum_{i=0}^{N-1} a_{i} f_{i}$. If $F$ is a $T$ superadditive process, a sequence $\mathbf{n}=\left\{n_{k}\right\}$ or a weight $\mathbf{a}=\left\{a_{i}\right\}$ is called good in the p-mean (a.e.) for $F$ if the limit of the associated averages exists in $L_{p}$-norm (a.e.).

2. Wiener-Wintner theorem for admissible processes. The particular class of superadditive processes we will study are the admissible processes. A family $\left\{f_{n}\right\}_{n \geq 0} \subset L_{p}$ is said to be $T$-admissible (or simply admissible) if $T f_{i} \leq f_{i+1}$ for $i \geq 0$. If $F=\left\{f_{n}\right\}$ is a $T$-admissible family, then the associated $T$-superadditive process $\left\{F_{n}\right\}_{n \geq 1}$, where $F_{n}=\sum_{i=0}^{n-1} f_{i}$, is called T-admissible. An admissible process $F$ is called strongly p-bounded (or simply strongly bounded when $p=1$ ) if $\sup _{n}\left\|f_{n}\right\|_{p}<\infty$.

When $p=1$, the boundedness and admissibility of the process $F$ implies that it is strongly bounded $[\mathrm{CF}]$. Therefore, strong boundedness and bound- 
edness are the same for admissible processes in $L_{1}$. Clearly, any strongly $p$-bounded process is $p$-bounded, however, when $p>1$, the converse is not the case. Indeed, as the following example shows, one can have an admissible 2-bounded process $F \subset L_{2}$ which is not strongly 2-bounded.

EXAMPLE. Let bounded positive functions $g_{n}, n=0,1,2, \ldots$, be given on some probability space, and suppose that a measure preserving point transformation $T$ is given such that the entire doubly-indexed family $\left\{T^{n} g_{m}\right\}_{n, m=0,1,2, \ldots}$ is independent, considered as a family of random variables. For example, we could take $T$ to be the product of countably many shifts on countably many infinite product spaces, and choose $g_{n}$ as a function of the first coordinate of the $n$th product space. Consider the case in which $g_{0}=0$ and $\int g_{n}=1 / 2^{n}, \int g_{n}^{2}=1 / \sqrt{n}$. Define $f_{n}, n=0,1,2, \ldots$, by

$$
f_{n}=T^{n} g_{0}+T^{n-1} g_{1}+\cdots+T g_{n-1}+g_{n} .
$$

As usual, define $F_{n}=f_{0}+f_{1}+\cdots+f_{n-1}$. It is easy to check that the sequence $\left\{f_{n}\right\}$ is $T$-admissible. Clearly $\int f_{n} \leq 1$ for all $n$. Also, since $\operatorname{Var}\left(g_{n}\right) \leq \int g_{n}^{2}$, independence yields

$$
\int f_{n}^{2} \leq\left(\int f_{n}\right)^{2}+\int g_{0}^{2}+\int g_{1}^{2}+\cdots+\int g_{n}^{2}
$$

and therefore,

$$
\int f_{n}^{2} \leq 1+c_{1} \sqrt{n}
$$

The independence assumption implies that $\left\{f_{i}\right\}$ is an independent family. It follows that

$$
\int F_{n}^{2} \leq\left(\int F_{n}\right)^{2}+\int f_{0}^{2}+\cdots+\int f_{n-1}^{2} \leq n^{2}+c_{2} n \sqrt{n},
$$

and so $\sup _{n} n^{-1}\left\|F_{n}\right\|_{2}<\infty$. But

$$
\int f_{n}^{2} \geq \int g_{0}^{2}+\int g_{1}^{2}+\cdots+\int g_{n}^{2} \geq c_{3} \sqrt{n}
$$

and hence $F$ is not strongly 2-bounded.

It is well known that every bounded superadditive process $F \subset L_{1}$ has an exact dominant [AS], that is, there exists a function $\delta \in L_{1}^{+}$such that $F_{n} \leq \sum_{i=0}^{n-1} T^{i} \delta$ for all $n \geq 1$ and $\int \delta=\sup _{n \geq 1} n^{-1}\left\|F_{n}\right\|_{1}$. It turns out that more is true for strongly $p$-bounded admissible processes $F \subset L_{p}$, $1 \leq p<\infty$.

Proposition 2.1. For any positive, strongly p-bounded T-admissible process $F=\left\{f_{n}\right\} \subset L_{p}, 1 \leq p<\infty$, there exists $\delta \in L_{p}^{+}$such that $f_{n} \leq T^{n} \delta$ for all $n \geq 0$ and $\|\delta\|_{p}=\sup _{n \geq 1}\left\|f_{n}\right\|_{p}=\lim _{n}\left\|f_{n}\right\|_{p}$.

Proof. Define a sequence $\left\{v_{n}\right\} \subset L_{p}^{+}$by $v_{n}=T^{-n} f_{n}, n \geq 0$. From the $T$-admissibility of $F$, we see that $v_{n} \leq v_{n+1}$. Thus, since $F$ is strongly 
$p$-bounded, by the monotone convergence theorem, $\delta:=\lim _{n} v_{n} \in L_{p}^{+}$and $\|\delta\|_{p}=\lim _{n}\left\|v_{n}\right\|_{p}$. Clearly, for $n \geq 0, v_{n} \leq \delta$, and hence $f_{n} \leq T^{n} \delta$.

Remarks. 1. The function $\delta \in L_{p}^{+}$is called a dominant for $F$. Clearly, if $p=1$, then $\delta$ is an exact dominant. Furthermore, if $b_{k}=\delta-v_{k}$, then $b_{k} \in L_{p}$ for each $k \geq 1$, and since $\left\|T^{k} \delta-f_{k}\right\|_{p} \rightarrow 0$, we have $\left\|b_{k}\right\|_{p} \rightarrow 0$ as $k \rightarrow \infty$, for all $1 \leq p<\infty$.

2. If the admissible process $F=\left\{f_{n}\right\}$ is not necessarily positive then the process $F^{\prime}=\left\{f_{n}^{\prime}\right\}$, where $f_{n}^{\prime}=f_{n}-T^{n} f_{0}$, as defined in Section 1 , is positive. Hence, by Proposition 2.1 there exists $\delta^{\prime} \in L_{p}^{+}$such that $f_{n}^{\prime} \leq T^{n} \delta^{\prime}$ for all $n \geq 0$ and $\left\|\delta^{\prime}\right\|_{p}=\lim _{n}\left\|f_{n}^{\prime}\right\|_{p}$. Therefore, the function $\delta=\delta^{\prime}+f_{0}$ satisfies $f_{n} \leq T^{n} \delta$ for all $n \geq 0$ and $\|\delta\|_{p}=\lim _{n}\left\|f_{n}\right\|_{p}$, which extends Proposition 2.1 to all strongly $p$-bounded admissible processes.

In order to study norm and a.e. convergence of the averages weighted by means of sequences induced by admissible processes, one should check whether such sequences have the right properties, like being Hartman almost periodic sequences to start with. Now we state and prove the WienerWintner type theorem for admissible processes, which shows that sequences induced by admissible processes indeed define Hartman almost periodic sequences.

TheOrem 2.2. Let $F=\left\{f_{n}\right\} \subset L_{1}$ be a bounded T-admissible process. There exists a set $N \in \Sigma$ with $\mu(N)=0$ such that for $x \in X \backslash N$,

$$
\frac{1}{n} \sum_{i=0}^{n-1} \lambda^{i} f_{i}(x) \quad \text { converges for all } \lambda \in \mathbb{K},
$$

where $\mathbb{K}$ is the unit circle in the complex plane.

Proof. The proof will be given for ergodic $T$, but it is also valid when $T$ is an arbitrary MPT by ergodic decomposition. Since the assertion is true for additive processes, by the Wienner-Wintner theorem, we can assume (by passing to $F^{\prime}$ if necessary) that $f_{i} \geq 0$ for each $i \geq 1$. Fix $k \in \mathbb{Z}^{+}$, and define

$$
g_{i}^{k}(x)= \begin{cases}T^{i-k} f_{k}(x) & \text { for } i>k, \\ f_{i}(x) & \text { for } 0 \leq i \leq k .\end{cases}
$$

Using the same notation as in Proposition 2.1 (and its proof), it follows that

$$
\begin{array}{cc}
0 \leq T^{i}\left(v_{i}-v_{k}\right) \leq f_{i}-g_{i}^{k} \leq T^{i} b_{k} & \text { if } i>k, \\
f_{i}-g_{i}^{k}=0 & \text { if } i \leq k,
\end{array}
$$

where $b_{k}=\delta-v_{k}$. Therefore $\sum_{i=0}^{n-1}\left(f_{i}-g_{i}^{k}\right) \leq \sum_{i=0}^{n-1} T^{i} b_{k}$. Because $v_{k} \uparrow \delta$, we have $\left\|b_{k}\right\|_{1}=\left\|\delta-v_{k}\right\|_{1} \downarrow 0$ as $k \rightarrow \infty$. 
Now, for each $n>k$ and $\lambda \in \mathbb{K}$, we have

$$
\sum_{i=0}^{n-1} \lambda^{i} g_{i}^{k}=\sum_{i=0}^{k-1} \lambda^{i} f_{i}+\sum_{i=k}^{n-1} \lambda^{i} T^{i-k} f_{k}=\sum_{i=0}^{k-1} \lambda^{i} f_{i}+\lambda^{k} \sum_{i=0}^{n-k-1} \lambda^{i} T^{i} f_{k} .
$$

Hence, by the Wiener-Wintner theorem, there exists a set $N_{k} \in \Sigma$, depending on $f_{k}$ only, with $\mu\left(N_{k}\right)=0$, such that for $x \notin N_{k}$,

$$
\lim _{n} \frac{1}{n} \sum_{i=0}^{n-1} \lambda^{i} g_{i}^{k}(x) \quad \text { exists for all } \lambda \in \mathbb{K} .
$$

Let $N^{\prime}=\bigcup_{k \geq 0} N_{k}$. Then $\mu\left(N^{\prime}\right)=0$, and for $x \notin N^{\prime}$,

$$
\lim _{n} \frac{1}{n} \sum_{i=0}^{n-1} \lambda^{i} g_{i}^{k}(x) \quad \text { exists for all } \lambda \in \mathbb{K} \text { and all } k \geq 0 \text {. }
$$

Next, observe that, by Birkhoff's individual ergodic theorem,

$$
\lim _{n} \frac{1}{n} \sum_{i=0}^{n-1} T^{i} b_{k}(x)=\int b_{k} \quad \text { for almost every } x .
$$

Let $M \in \Sigma$ be the set with $\mu(M)=0$ such that for $x \notin M$,

$$
\lim _{n} \frac{1}{n} \sum_{i=0}^{n-1} T^{i} b_{k}(x)=\int b_{k}
$$

for all $k \geq 0$. If $N=M \cup N^{\prime}$, then $\mu(N)=0$. Furthermore, if $\mathbf{a}=\left\{a_{i}\right\}$ and $\mathbf{a}^{k}=\left\{a_{i}^{k}\right\}$, where $a_{i}=f_{i}(x)$ and $a_{i}^{k}=g_{i}^{k}(x)$ for a fixed $x \in X \backslash N$, then

$$
\begin{aligned}
0 & \leq\left\|\mathbf{a}-\mathbf{a}^{k}\right\|_{1}=\underset{n}{\limsup } \frac{1}{n} \sum_{i=0}^{n-1}\left|a_{i}-a_{i}^{k}\right|=\underset{n}{\limsup } \frac{1}{n} \sum_{i=0}^{n-1}\left|f_{i}(x)-g_{i}^{k}(x)\right| \\
& \leq \underset{n}{\limsup } \frac{1}{n} \sum_{i=0}^{n-1} T^{i} b_{k}(x)=\int b_{k} d \mu=\left\|b_{k}\right\|_{1} \downarrow 0 \quad \text { as } k \rightarrow \infty .
\end{aligned}
$$

Hence $\left\|\mathbf{a}-\mathbf{a}^{k}\right\|_{1} \rightarrow 0$. Therefore, by Lemma 3.1 of [BO] (or Lemma 2.2(b) of [JO]), $n^{-1} \sum_{i=0}^{n-1} \lambda^{i} f_{i}(x)$ converges for all $\lambda \in \mathbb{K}$.

REMARK. The limit in Theorem 2.2 is 0 for all $\lambda \neq 1$ if $T$ is weakly mixing $[\mathrm{BeL}]$.

3. Weights induced by admissible processes. An important consequence of Theorem 2.2 is that, for a.e. $x$, the sequence $\left\{f_{i}(x)\right\}$, where $F=\left\{f_{i}\right\} \subset L_{p}$ is a strongly $p$-bounded admissible superadditive process, has Fourier coefficients, i.e., is a Hartman almost periodic sequence [LOT]. Furthermore, by the a.e. convergence of the averages of such processes [C]], it is easy to see that $\left\{f_{i}(x)\right\} \in W_{p}, 1 \leq p<\infty$, where $W_{p}:=\left\{\mathbf{a}=\left\{a_{i}\right\}\right.$ : 
$\left.\|\mathbf{a}\|_{W_{p}}^{p}=\lim \sup _{n} n^{-1} \sum_{i=0}^{n-1}\left|a_{i}\right|^{p}<\infty\right\}$, and $W_{\infty}$ is the class of bounded sequences. It is known that, even in the additive case, a Hartman sequence need not be a good sequence for a.e. convergence [LOT]. On the other hand, if $F \subset L_{\infty}$ is a 1-bounded admissible process, by the observations above and Corollary 4.3 of [BeL], for a.e. $x,\left\{f_{i}(x)\right\}$ is a sequence good a.e. for any dynamical system $\left(\Omega, \Sigma^{\prime}, \nu, S\right)$, where $S$ has Lebesgue spectrum. Thus, it is natural to ask whether the sequences of weights induced by admissible superadditive processes are good a.e. or in the mean in $L_{p}$. Now we turn to the study of this question.

Theorem 3.1. Let $(X, \Sigma, \mu)$ be a Lebesgue space and let $F=\left\{f_{i}\right\} \subset L_{p}$, $1 \leq p \leq \infty$, be a strongly $p$-bounded T-admissible process. Let $q$ be the dual index. Then for a.e. $x \in X$, the sequence $\mathbf{a}=\left\{f_{i}(x)\right\}$ is good a.e. in $L_{q}$.

Proof. First, observe that if $\left\{f_{i}\right\}$ is not positive, then the process $\left\{f_{i}^{\prime}\right\}=$ $\left\{f_{i}-T^{i} f_{0}\right\}$ is a positive $T$-admissible strongly $p$-bounded process. By the return times theorem [Bo, $\mathrm{Ru}]$, for a.e. $x \in X$ the sequence $\left\{T^{i} f_{0}(x)\right\}$ is good a.e. in $L_{q}$. Hence, without loss of generality, we can assume that the process $F$ is positive.

Now we will use the same machinery as in Theorem 2.2. Define $\mathbf{a}^{k}=$ $\left\{a_{i}^{k}\right\}_{i=0}^{\infty}, k \geq 0$, as $a_{i}^{k}=g_{i}^{k}(x)$. First, assume that $F \subset L_{\infty}$. Then there is a null set $N \subset X$ such that for $x \notin N$, the sequence $\mathbf{a}^{k}$ is good a.e. in $L_{1}$ for all $k \geq 0[\mathrm{Bo}, \mathrm{Ru}]$. For such an $x$, if $\mathbf{a}=\left\{f_{i}(x)\right\}$, then

$$
\begin{aligned}
0 \leq\left\|\mathbf{a}^{k}-\mathbf{a}\right\|_{W_{\infty}} & \leq \sup _{i}\left|T^{i}\left(\delta-v_{k}\right)(x)\right| \leq \sup _{i}\left\|T^{i}\left(\delta-v_{k}\right)\right\|_{L_{\infty}(X)} \\
& \leq\left\|\left(\delta-v_{k}\right)\right\|_{L_{\infty}(X)} \quad \text { since } T \text { is a contraction on } L_{\infty} .
\end{aligned}
$$

Since $v_{k} \uparrow \delta$ a.e., we have $\lim _{k}\left\|\mathbf{a}^{k}-\mathbf{a}\right\|_{W_{\infty}} \leq \lim _{k}\left\|\delta-v_{k}\right\|_{L_{\infty}}=0$. Now, let $(\Omega, \Sigma, \nu, S)$ be a dynamical system and $g \in L_{\infty}(\Omega)$. Then

$$
\begin{aligned}
\mid \frac{1}{n} \sum_{i=0}^{n-1} a_{i} & S^{i} g(\omega)-\frac{1}{m} \sum_{i=0}^{m-1} a_{i} S^{i} g(\omega)|\leq| \frac{1}{n} \sum_{i=0}^{n-1}\left(a_{i}-a_{i}^{k}\right) S^{i} g(\omega) \mid \\
+ & \left|\frac{1}{n} \sum_{i=0}^{n-1} a_{i}^{k} S^{i} g(\omega)-\frac{1}{m} \sum_{i=0}^{m-1} a_{i}^{k} S^{i} g(\omega)\right|+\left|\frac{1}{m} \sum_{i=0}^{m-1}\left(a_{i}-a_{i}^{k}\right) S^{i} g(\omega)\right| \\
\leq & \left\|\mathbf{a}-\mathbf{a}^{k}\right\|_{W_{\infty}}\left[\frac{1}{n} \sum_{i=0}^{n-1} S^{i}|g(\omega)|+\frac{1}{m} \sum_{i=0}^{m-1} S^{i}|g(\omega)|\right] \\
+ & \left|\frac{1}{n} \sum_{i=0}^{n-1} a_{i}^{k} S^{i} g(\omega)-\frac{1}{m} \sum_{i=0}^{m-1} a_{i}^{k} S^{i} g(\omega)\right| .
\end{aligned}
$$

Since $\left\|\mathbf{a}^{k}-\mathbf{a}\right\|_{W_{\infty}} \rightarrow 0$, and since $n^{-1} \sum_{i=0}^{n-1} S^{i}|g|$ converges a.e. to a function $g^{*}$ with $\int\left|g^{*}\right|<\infty$, it follows that $\left\{n^{-1} \sum_{i=0}^{n-1} a_{i} S^{i} g(\omega)\right\}$ is Cauchy, hence 
converges a.e. Thus, by [LOT, Proposition 3.1], $n^{-1} \sum_{i=0}^{n-1} a_{i} S^{i} g$ converges a.e. for all $g \in L_{1}(\Omega)$.

For $F \subset L_{p}, 1 \leq p<\infty$, we adopt the method of proof of [LOT, Proposition 3.2]. By [LOT, Theorem 3.6], for each $k, \mathbf{a}^{k}$ is good a.e. in $L_{q}$, hence in $L_{\infty}$. Again, if $(\Omega, \Sigma, \nu, S)$ is a dynamical system and $f \in L_{\infty}(\Omega)$, then for a.e. $\omega \in \Omega$,

$$
\begin{aligned}
\mid \frac{1}{n} \sum_{i=0}^{n-1} a_{i} & S^{i} f(\omega)-\frac{1}{m} \sum_{i=0}^{m-1} a_{i} S^{i} f(\omega)|\leq| \frac{1}{n} \sum_{i=0}^{n-1}\left(a_{i}-a_{i}^{k}\right) S^{i} f(\omega) \mid \\
+ & \left|\frac{1}{n} \sum_{i=0}^{n-1} a_{i}^{k} S^{i} f(\omega)-\frac{1}{m} \sum_{i=0}^{m-1} a_{i}^{k} S^{i} f(\omega)\right|+\left|\frac{1}{m} \sum_{i=0}^{m-1}\left(a_{i}-a_{i}^{k}\right) S^{i} f(\omega)\right| \\
\leq & \|f\|_{\infty}\left[\frac{1}{n} \sum_{i=0}^{n-1}\left|a_{i}-a_{i}^{k}\right|+\frac{1}{m} \sum_{i=0}^{m-1}\left|a_{i}-a_{i}^{k}\right|\right] \\
+ & \left|\frac{1}{n} \sum_{i=0}^{n-1} a_{i}^{k} S^{i} f(\omega)-\frac{1}{m} \sum_{i=0}^{m-1} a_{i}^{k} S^{i} f(\omega)\right| .
\end{aligned}
$$

Since $\left\|\mathbf{a}^{k}-\mathbf{a}\right\|_{W_{1}} \leq\left\|b_{k}\right\|_{1} \rightarrow 0$, we conclude that $\left\{n^{-1} \sum_{i=0}^{n-1} a_{i} S^{i} f(\omega)\right\}$ is Cauchy, hence converges. Since $\left\{f_{i}(x)\right\} \in W_{p}$ is good a.e. in $L_{\infty}$, [LOT, Proposition 3.1] shows that $\left\{f_{i}(x)\right\}$ is good a.e. in $L_{q}$.

Remarks. 1. Theorem 3.1 extends the return times theorem to sequences generated by admissible processes.

2. Theorem 2.2 follows from Theorem 3.1 (use all possible rotations since a 1-bounded admissible process in $L_{1}$ is strongly bounded [ÇF]).

The fact that the sequences considered in Theorem 3.1 are good for the norm convergence follows from the method employed in the previous theorem and [ÇLO, Theorem 4.3] when $p=1$, and [ÇLO, Theorem 4.2] when $1<p<\infty$ :

Proposition 3.2. Let $F=\left\{f_{i}\right\} \subset L_{p}, 1 \leq p<\infty$, be a strongly $p$ bounded admissible process. Then for almost every $x$ the sequence $\left\{f_{i}(x)\right\}$ is good in the p-mean.

Theorem 3.1 can also be interpreted as stating that if $(X, \Sigma, \mu)$ is a Lebesgue space, $T: X \rightarrow X$ is an invertible MPT, and $F \subset L_{p}, 1 \leq p \leq \infty$, is a strongly $p$-bounded $T$-admissible process, then there exists a set $N \in \Sigma$ with $\mu(N)=0$ such that for any $x \in X \backslash N$ the sequence $\left\{f_{i}(x)\right\}$ is good a.e. for $L_{q}$, where $q$ is the dual index. This fact, combined with [Ç, Theorem 3.1], leads to a further generalization of the return times theorem: 
Theorem 3.3. Let $(X, \Sigma, \mu)$ be a Lebesgue space and $T: X \rightarrow X$ be an invertible $M P T$. If $F \subset L_{p}, 1 \leq p \leq \infty$, is a strongly $p$-bounded T-admissible process, then there exists a set $N \in \Sigma$ with $\mu(N)=0$ such that for any $x \in X \backslash N$ the sequence $\left\{f_{i}(x)\right\}$ is good a.e. for any strongly q-bounded admissible process (in $L_{q}$ ) relative to invertible MPTs, where $q$ is the dual index.

As observed in [LOT, Proposition 1.5], a sequence induced by an additive process need not be a Besicovitch sequence. Hence, a sequence $\left\{f_{i}(x)\right\}$ induced by an admissible superadditive process need not be a Besicovitch sequence either. Indeed, $\left\{f_{i}(x)\right\}$ is a linear combination of a Besicovitch sequence, a shift orthogonal sequence and a sequence with zero mean. However, with an additional condition on $T$ one obtains Besicovitch sequences induced by admissible processes.

Theorem 3.4. Let $\left\{f_{i}\right\} \subset L_{\infty}^{+}$be a 1-bounded T-admissible process. Assume that $T$ has discrete spectrum. Then for a.e. $x$, the sequence of weights $\left\{f_{i}(x)\right\}$ is bounded Besicovitch.

Proof. Define sequences $\left\{\mathbf{s}^{k}\right\}$ and $\mathbf{s}$ by $s_{i}^{k}=g_{i}^{k}(x)$ and $s_{i}=f_{i}(x)$ for all $i \geq 1$, where $g_{i}^{k}$ is as defined in the proof of Theorem 2.2. For each $k$, there is $N_{k} \subset X$ with $\mu\left(N_{k}\right)=0$ such that $\mathbf{s}^{k}$ is bounded Besicovitch [BeL, Theorem 3.22]. Obviously, $\left\|\mathbf{s}^{k}\right\|_{\infty} \leq\|\mathbf{s}\|_{\infty}$. As in the proof of Theorem 2.2,

$$
\left\|\mathbf{s}^{k}-\mathbf{s}\right\|_{W_{1}}=\limsup _{n} \frac{1}{n} \sum_{i=0}^{n-1}\left|s_{i}^{k}-s_{i}\right| \leq \limsup _{n} \frac{1}{n} \sum_{i=0}^{n-1} T^{i} b_{k}=\left\|b_{k}\right\|_{1} \downarrow 0 .
$$

Thus, $\mathbf{s}$ is in the sup-dominated $W_{1}$-closure of the space of bounded Besicovitch sequences. Hence by Lemma 3.23 of Baxter-Olsen [BO], for any fixed $x \in \bigcap N_{k}^{\mathrm{c}}$ the sequence $\mathbf{s}$ is bounded Besicovitch.

Next, we will introduce a class of good sequences of weights induced by admissible processes and investigate some interesting properties of such weights. To do so, in the following definition and in Theorem 3.5 below we will assume that $(X, \Sigma, \mu, T)$ is a strictly L-stable system $[\mathrm{BrK}]$, where $X$ is a compact connected metric space, and $\left\{v_{i}\right\} \subset L_{\infty}^{+}$is an increasing sequence of Riemann integrable functions on $X$ with $v_{i} \uparrow v$ uniformly.

Definition. For any $x \in X$ the sequence $\mathbf{a}=\left\{a_{k}\right\}$, where $a_{k}=$ $v_{k}\left(T^{k} x\right)$, is called near-uniform.

Remarks. 1. Every uniform sequence is near-uniform. To see this, let $v_{i}=\chi_{Y}$, where $(X, \Sigma, m, T, Y)$ is the apparatus for the uniform sequence $[\mathrm{BrK}]$.

2. By Theorem 3.1, for a.e. $x \in X$, near-uniform sequences are good a.e. in $L_{1}$. 
Theorem 3.5. Near-uniform sequences are good a.e. in $L_{1}$ for all $x \in X$.

Proof. By the method of proof of Theorem 3.1, all one needs to show is that

$$
\lim _{n} \frac{1}{n} \sum_{i=0}^{n-1} f_{i}(x) \quad \text { exists for all } x \in X,
$$

where $f_{i}=T^{i} v_{i}$. By the uniform convergence, $v$ is necessarily Riemann integrable, and $\gamma_{F}=\|v\|_{1}$. Given $\varepsilon>0$, find continuous functions $r$ and $s$ such that

$$
0 \leq r \leq v \leq s \quad \text { for every } x \in X, \quad \text { and } \quad \int(s-r)<\varepsilon .
$$

Furthermore, we can assume that, for large enough $K$, if $k \geq K$, we have $r \leq v_{k}$. Defining $g_{i}^{k}$ as before, for $k \geq K$, we see that $n^{-1} \sum_{i=0}^{n-1} g_{i}^{k} \leq$ $n^{-1} \sum_{i=0}^{n-1} f_{i}(x)$. Since $T^{i} r \leq T^{i} v_{k}$ for all $i \geq 0$, we have $T^{i} r \leq T^{i-k} f_{k}$. Similarly, $v_{i} \leq s$ for all $i$ implies that $f_{i} \leq T^{i} s$ for all $i$. Hence, by ignoring $\sum_{i=0}^{k-1} g_{i}^{k}$ if necessary, we have

$$
0 \leq \frac{1}{n} \sum_{i=0}^{n-1} T^{i} r \leq \frac{1}{n} \sum_{i=0}^{n-1} g_{i}^{k} \leq \frac{1}{n} \sum_{i=0}^{n-1} f_{i} \leq \frac{1}{n} \sum_{i=0}^{n-1} T^{i} s .
$$

Since $T$ is $L$-stable, both $\lim n^{-1} \sum_{i=0}^{n-1} T^{i} r(x)=\int r$ and $\lim n^{-1} \sum_{i=0}^{n-1} T^{i} s(x)$ $=\int s$ exist for every $x \in X$. Hence, for every $x \in X$,

$$
\begin{aligned}
0 \leq \limsup \frac{1}{n} \sum_{i=0}^{n-1} f_{i}(x)-\liminf \frac{1}{n} \sum_{i=0}^{n-1} f_{i}(x) & \leq \lim \frac{1}{n} \sum_{i=0}^{n-1} T^{i}(s-r)(x) \\
& =\int(s-r)<\varepsilon .
\end{aligned}
$$

Hence $\lim n^{-1} \sum_{i=0}^{n-1} f_{i}(x)$ exists for all $x \in X$.

Remark. Let $\left\{A_{n}\right\}$ and $A$ be measurable with $A_{n} \uparrow A$ such that $\sum \mu\left(A_{n} \backslash A_{n-1}\right)<\infty$. Then, letting $v_{i}=\chi_{A_{i}}$ and $v=\chi_{A}$, the observations above imply immediately that the "return-time sequence" $\left\{n_{k}\right\}$ defined by

$$
n_{k}=\inf \left\{l \in \mathbb{Z}: T^{l}(x) \in A_{k}, l \geq n_{k-1}\right\}
$$

is good a.e. in $L_{1}$. Also, it follows that the frequency of the relation $T^{n} x \in A_{n}$ exists a.e. and is equal to $\mu(A)$ iff $T$ is ergodic.

4. Purely subadditive parts of admissible processes. By the "decomposition theorem" of Kingman any bounded $T$-superadditive process $F=\left\{F_{n}\right\} \subset L_{1}$ can be decomposed into a difference $G-H$, where $G=\left\{G_{n}\right\}$ is an additive process and $H=\left\{H_{n}\right\}$ is a positive subadditive process [AS]. In fact, $H$ is a purely subadditive process, in the sense that it does not dominate any nonzero $T$-additive process and $\lim _{n} n^{-1} H_{n}=0$ a.e. (and hence 
in norm) [AS]. Furthermore, $G_{n}=\sum_{k=0}^{n-1} T^{k} \delta$, where $\delta$ is an exact dominant of $F$. If $H=\left\{H_{n}\right\}$ is the purely subadditive part of a superadditive process, then necessarily $H_{n}=\sum_{i=0}^{n-1} h_{i} \geq 0$ for each $n \geq 0$, but this does not imply that each $h_{i} \geq 0$. However, if $F$ is admissible, then by Proposition 2.1 (and with the same notation as in its proof), $h_{i}=T^{i} \delta-f_{i} \geq 0$. Also, $\left\|h_{i}\right\|_{1}=\left\|\delta-v_{i}\right\|_{1}$ for each $i \geq 0$. Hence we have

Proposition 4.1. If $H=\left\{H_{n}\right\} \subset L_{1}$ is the purely subadditive part of a $T$-admissible process, where $H_{n}=\sum_{i=0}^{n-1} h_{i}$, then $h_{i} \geq 0$ for all $i \geq 0$.

The limit in Theorem 2.2 is identified (as 0 for $\lambda \neq 1$ ) if $T$ is weakly mixing. For more general cases of weights involving admissible processes, one utilizes the positivity of the purely subadditive part of an admissible process and the following observation.

Proposition 4.2. If $H=\left\{h_{n}\right\} \subset L_{1}$ is the purely subadditive part of a T-admissible process and $\mathbf{a} \in l_{\infty}$ is a sequence which is good a.e., then $\lim _{n} n^{-1} \sum_{k=0}^{n-1} a_{k} h_{k}=0$ a.e. and in norm.

Proof. If $\mathbf{a}$ is good a.e., then by [Ç, Theorem 3.1] it is good a.e. for any bounded admissible process $F \subset L_{1}$. In particular, if $H=\left\{h_{n}\right\}$ is the purely subadditive part of $F$, then $\lim _{n} n^{-1} \sum_{k=0}^{n-1} a_{k} h_{k}$ exists a.e. Furthermore (see $[\mathrm{AS}])$,

$$
0 \leq \lim _{n} \frac{1}{n}\left|\sum_{k=0}^{n-1} a_{k} h_{k}\right| \leq \lim _{n} \frac{1}{n} \sum_{k=0}^{n-1} h_{k}=0 \quad \text { a.e. and in norm. }
$$

REMARKS. 1. If $\mathbf{a} \in l_{\infty}$ is a sequence which is good a.e. or good in the 1-mean (in particular, $a_{n}=\lambda^{n}, \lambda \in \mathbb{C}$ ), then for any bounded $T$-admissible process $F \subset L_{1}$,

$$
\lim _{n} \frac{1}{n} \sum_{k=0}^{n-1} a_{k} f_{k}=\lim _{n} \frac{1}{n} \sum_{k=0}^{n-1} a_{k} T^{k} \delta,
$$

where $\delta$ the exact dominant for $F$.

2. In [LOT] a series representation of the limit of weighted averages of additive processes in $L_{p}, 1 \leq p<\infty$, has been obtained. Proposition 4.2, combined with their results and the remark above, also yields a series representation of the limit of weighted averages for admissible processes.

3. It is known that the spectrum $\sigma(\mathbf{a})=\{\lambda \in \mathbb{C}:|\lambda|=1, c(\lambda) \neq 0\}$ of a Hartman sequence a is countable [Ka]. If $T$ is invertible and $F$ is a 1-bounded admissible process with purely subadditive part $H=\left\{h_{k}\right\}$, then by Proposition $4.1, \lim _{n} n^{-1} \sum_{k=0}^{n-1} \lambda^{k} h_{k}=0$ a.e., and hence

$$
c(\lambda)=\lim _{n} \frac{1}{n} \sum_{k=0}^{n-1} \lambda^{k} f_{k}=\lim _{n} \frac{1}{n} \sum_{k=0}^{n-1} \lambda^{k} T^{k} \delta .
$$


So, the spectrum of the weights induced by $F$ is the same as the spectrum of the weights induced by the additive process defined by its exact dominant. Consequently, following the arguments in [LOT, Theorem 3.15], the limit in Theorem 3.1 is equal to the limit of the additive part.

We close this section with the following observation on the behavior of the purely subadditive part of an admissible process. Recall that, if a sequence $\left\{a_{i}\right\}$ has mean zero (i.e. $\lim _{n} n^{-1} \sum_{k=0}^{n-1} a_{i}=0$ ), then there exists a set $K \subset \mathbb{N}$ of zero density such that $\lim _{n \in \mathbb{N} \backslash K, n \rightarrow \infty} a_{n}=0$. So, for a fixed $x \in X$, since $\lim _{n} n^{-1} \sum_{k=0}^{n-1} h_{i}=0$, there exists a set $K \subset \mathbb{N}$ of density 0 such that $\lim _{n \in \mathbb{N} \backslash K, n \rightarrow \infty} h_{n}(x)=0$. On the other hand, since $H_{n} \leq 0$, we also have

$$
0 \leq \int \frac{1}{n} H_{n}=\frac{1}{n} \int \sum_{k=0}^{n-1} h_{i}=\frac{1}{n} \sum_{k=0}^{n-1} \int h_{i} .
$$

By subadditivity, $H_{n} \leq \sum_{k=0}^{n-1} T^{i} h_{0}$, which implies that

$$
0 \leq \frac{1}{n} H_{n} \leq \frac{1}{n} \sum_{k=0}^{n-1} T^{i} h_{0}
$$

and the right hand side converges, so is bounded. Hence the sequence $\left\{n^{-1} H_{n}\right\}$ is bounded. Now, if $g_{n}=n^{-1} \sum_{k=0}^{n-1} T^{i} h_{0}$, then $n^{-1} H_{n} \leq g_{n}$, and $g_{n} \rightarrow g^{*}$ a.e. for some $g^{*} \in L_{1}$. Since $n^{-1} H_{n} \rightarrow 0$ a.e., by the generalized Lebesgue convergence theorem, $\lim \int g_{n} \rightarrow \int g^{*}$ implies that $0=$ $\lim _{n} n^{-1} \int H_{n}=\lim _{n} n^{-1} \sum_{k=0}^{n-1} \int h_{i}$. Consequently, there exists a set $K \subset \mathbb{N}$ of density 0 such that $\lim _{n \in \mathbb{N} \backslash K, n \rightarrow \infty} \int h_{n}(x)=0$. Since, by admissibility, $h_{m} \leq T^{k} h_{m-k}$ for any $m \geq k \geq 0$, we must have $\lim _{n \rightarrow \infty} \int h_{n}(x)=0$ while $n$ ranges through all positive integers.

\section{Norm convergence of averages along generalized Besicovitch} weights. In what follows we will still consider a probability space $(X, \Sigma, \mu)$ and a measure preserving transformation $T: X \rightarrow X$, not necessarily invertible. We will also consider the group $U$ of all unimodular functions on $X$ :

$$
U=\left\{u \in L_{\infty}(X):|u(x)|=1 \text { a.e. on } X\right\} .
$$

One can deduce from the Wiener-Wintner theorem that for every $f \in L_{1}$ there exists a null set $N$ such that for $x \notin N$ and any $u \in U$ the averages

$$
\frac{1}{n} \sum_{k=0}^{n-1} u^{k}(x) f\left(T^{k} x\right)
$$

converge whenever $|u(x)|=1$. This observation naturally motivates the question of whether it is possible to obtain the norm and a.e. convergence 
of the averages of the form

$$
\frac{1}{n} \sum_{k=0}^{n-1} u^{k}\left(T^{k} x\right) f\left(T^{k} x\right)=\frac{1}{n} \sum_{k=0}^{n-1}\left[\left(u^{k} f\right) \circ T^{k}\right](x)
$$

if $u \in U$ and $f \in L_{1}(X)$.

In this section we shall examine the norm convergence of the averages $(*)$. Note that, due to the nature of these averages, a standard argument with the telescoping sum effect for a uniformly bounded transformation in a Banach space appears to be of no help. Still, it would be desirable to obtain the $L_{1}$-norm convergence of the averages $(*)$ for all $u \in U$ and $f \in L_{1}$. The argument in this section will allow us to show that the norm convergence takes place when $u \in U$ assumes countably many values; in this case we will write $u \in U_{\mathrm{c}}$. Define

$$
U_{1}=\left\{u \in U:\left\{u^{k}: k \in \mathbb{Z}\right\} \text { is precompact in } L_{1}\right\} .
$$

As is seen below the class $U_{1}$ is not empty. In fact, Corollary 5.3 provides a method of constructing nontrivial representatives of $U_{1}$. At the same time, the next example indicates that $U_{1} \neq U$. The authors are thankful to the anonymous referee for this example.

EXAMPLE 5.1. If $X=\mathbb{K}$ with Lebesgue's measure, and $u(z)=z, z \in \mathbb{K}$, then $u \notin U_{1}$. In order to see this consider the sequence $u_{n}(z)=z^{n}, n=$ $1,2, \ldots$, and show that, given any $h \in L_{\infty}(\mathbb{K})$, we have $\int u_{n} h d \mu \rightarrow 0$ as $n \rightarrow \infty$. Indeed, for every positive integer $m$,

$$
\int z^{m} d \mu=\int_{0}^{1} e^{i t m} d t \rightarrow 0
$$

as $m \rightarrow \infty$, therefore, $\int u_{n} p d \mu \rightarrow 0$ for every polynomial $p$ on $\mathbb{K}$. Since the polynomials are dense in $L_{1}(\mathbb{K})$, we have $u_{n} \rightarrow 0$ weakly in $L_{1}$. Together with the facts that $U$ is closed in $L_{1}$ and $0 \notin U$, this implies that $\left\{u^{n}: n \in \mathbb{N}\right\}$ cannot have a subsequence converging in $L_{1}$, hence $\left\{u^{k}: k \in \mathbb{Z}\right\}$ is not precompact in $L_{1}$.

A sequence $\left\{E_{n}\right\}_{n=1}^{\infty} \subset \Sigma$ will be called a disjoint measurable partition (d.m.p.) of $X$ if $\mu\left(E_{n}\right)>0$ for each $n, X=\bigcup E_{n}$, and $E_{i} \cap E_{j}=\emptyset$ for $i \neq j$.

Lemma 5.2. Let $g_{n}: X \rightarrow \mathbb{K}$ be a sequence of measurable functions, and let $\left\{E_{i}\right\}$ be a d.m.p. of $X$ such that $\left.g_{n}\right|_{E_{i}} \equiv \lambda_{n i}=$ const for all $n, i=1,2, \ldots$. Then $\left\{g_{n}\right\}$ has a subsequence $\left\{g_{n_{m}}\right\}$ converging in $L_{1}$-norm.

Proof. We define the sets $G_{m}$ inductively as follows. Let $G_{0}=\left\{g_{n}\right\}$ and $I_{0}=\mathbb{N}$. Let $\nu_{i}$ be an accumulation point of the set $\left\{\lambda_{n i}\right\}_{n \in I_{m-1}}$, and let 


$$
\begin{aligned}
G_{m} & =\bigcap_{i=1}^{m}\left\{g_{n} \in G_{m-1}:\left|\nu_{i}-\lambda_{n i}\right|<\left[2^{i} m \mu\left(E_{i}\right)\right]^{-1}\right\}, \\
I_{m} & =\left\{n: g_{n} \in G_{m}\right\}, \quad m=1,2, \ldots
\end{aligned}
$$

Define $g_{0}$ to be such that $\left.g_{0}\right|_{E_{m}} \equiv \nu_{m}, m=1,2, \ldots$, and pick $g_{n_{m}} \in G_{m}$, $m \geq 1$. Then we have

$$
\begin{aligned}
\left\|g_{0}-g_{n_{m}}\right\|_{1} & =\sum_{i=1}^{m} \int_{E_{i}}\left|g_{0}-g_{n_{m}}\right| d \mu+\sum_{i=m+1}^{\infty} \int_{E_{i}}\left|g_{0}-g_{n_{m}}\right| d \mu \\
& \leq \sum_{i=1}^{m} \int_{E_{i}}\left|\nu_{i}-\lambda_{n_{m} i}\right| d \mu+2 \sum_{i=m+1}^{\infty} \mu\left(E_{i}\right) \\
& <\frac{1}{m} \sum_{i=1}^{m} \frac{1}{2^{i}}+2 \sum_{i=m+1}^{\infty} \mu\left(E_{i}\right) \\
& <\frac{1}{m}+2 \sum_{i=m+1}^{\infty} \mu\left(E_{i}\right) \rightarrow 0 \quad \text { as } m \rightarrow \infty
\end{aligned}
$$

Corollary 5.3. $U_{\mathrm{c}} \subset U_{1}$.

REMARK. The authors do not know whether or not $U_{\mathrm{c}}=U_{1}$.

Now we shall state the following straightforward fact without proof.

Lemma 5.4. Let $(B,\|\cdot\|)$ be a Banach space, and let $\left\{A_{n}\right\} \subset B$ be a sequence such that for every $\varepsilon>0$ there is a convergent sequence $\left\{\widehat{A}_{n}\right\} \subset B$ and a positive integer $N$ for which

$$
\left\|A_{n}-\widehat{A}_{n}\right\|<\varepsilon \quad \text { for all } n \geq N .
$$

Then the sequence $\left\{A_{n}\right\}$ also converges in $B$.

Let $\mathbb{L}_{\infty}(X)$ denote the ${ }^{*}$-algebra of all measurable functions on $X$ (and not their equivalence classes). Since $\mu$ is finite, there exists a ${ }^{*}$-homomorphism $L_{\infty}(X) \ni g \mapsto \widetilde{g} \in \mathbb{L}_{\infty}(X)$ such that $\widetilde{1}(x)=1$ and $\widetilde{0}(x)=0$ for all $x \in X$. Such a homomorphism is called a lifting (see $[\mathrm{Ku}]$, for example).

THEOREM 5.5. For every $u \in U_{\mathrm{c}}$ and $f \in L_{\infty}$, the averages

$$
A_{n}(u ; f)=\frac{1}{n} \sum_{k=0}^{n-1}\left(u^{k} f\right) \circ T^{k}
$$

converge in $L_{p}$-norm for all $1 \leq p<\infty$.

Proof. We start with an adaptation of the scheme of the proof of Theorem 5 in $[\mathrm{Ry}]$. Let $[u]$ denote the $L_{1}$-closure of the group $\left\{u^{k}: k \in \mathbb{Z}\right\}$, which, by Corollary 5.3, is a compact group. Consider the Haar measure 
on $[u]$. Define

$$
X^{\prime}=X \otimes[u]
$$

and $T_{u}: X^{\prime} \rightarrow X^{\prime}$ by

$$
T_{u}(x, v)=(T x, u v) .
$$

By the invariance property of Haar measure, $T_{u}$ is a MPT on $X^{\prime}$. Let $L_{\infty} \ni$ $g \mapsto \widetilde{g} \in \mathbb{L}_{\infty}$ be a lifting. If we define $f^{\prime}$ on $X^{\prime}$ by $f^{\prime}(x, v)=(\widetilde{v} f)(x)=$ $\widetilde{v}(x) f(x)$, then $f^{\prime}$ is measurable by Theorem A.5 (see Appendix), so $f^{\prime} \in$ $L_{\infty}\left(X^{\prime}\right)$. By Birkhoff's individual ergodic theorem, the averages

$$
\begin{aligned}
\frac{1}{n} \sum_{k=0}^{n-1} f^{\prime}\left(T_{u}^{k}(x, v)\right) & =\frac{1}{n} \sum_{k=0}^{n-1} f^{\prime}\left(T^{k} x, u^{k} v\right)=\frac{1}{n} \sum_{k=0}^{n-1} \widetilde{\left(u^{k} v f\right)}\left(T^{k} x\right) \\
& =\frac{1}{n} \sum_{k=0}^{n-1}\left(u^{k} v f\right)\left(T^{k} x\right)
\end{aligned}
$$

converge for almost all $(x, v) \in X^{\prime}$. It follows from Fubini's theorem that, for almost all $v \in[u]$, the averages

$$
A_{n}(u, v ; f)=\frac{1}{n} \sum_{k=0}^{n-1}\left(u^{k} v f\right) \circ T^{k}
$$

converge a.e. on $X$. Since the $L_{\infty}$-norms of these averages are bounded (by $\|f\|_{\infty}$ ), the bounded convergence theorem yields $L_{p^{-}}$-convergence of $A_{n}(u, v ; f)$ for almost every $v \in[u]$.

Since the Haar measure of an open set is positive, any $L_{1}$-neighborhood of the unit $1 \in[u]$ contains a $v \in[u]$ for which $\left\{A_{n}(u, v ; f)\right\}$ converges in $L_{p}$. Therefore, for $\varepsilon>0$ one can find $v \in[u]$ such that

$$
\left\{A_{n}(u, v ; f)\right\} \text { converges in } L_{p} \text { and }\|1-v\|_{1}<2\left(\frac{\varepsilon}{2\|f\|_{\infty}}\right)^{p} .
$$

Then

$$
\begin{aligned}
\| A_{n}(u ; f)- & A_{n}(u, v ; f) \|_{p} \\
& =\left\|\frac{1}{n} \sum_{k=0}^{n-1}\left(u^{k} f-u^{k} v f\right) \circ T^{k}\right\|_{p} \leq \frac{1}{n} \sum_{k=0}^{n-1}\left\|u^{k} f-u^{k} v f\right\|_{p} \\
& \leq \frac{1}{n} \sum_{k=0}^{n-1}\left\|u^{k}\right\|_{\infty}\|f-v f\|_{p}=\|f-v f\|_{p} \leq\|1-v\|_{p}\|f\|_{\infty} \\
& \leq\|1-v\|_{\infty}^{(p-1) / p} \cdot\|1-v\|_{1}^{1 / p} \cdot\|f\|_{\infty} \\
& <2^{(p-1) / p} \cdot 2^{1 / p} \cdot \frac{\varepsilon}{2\|f\|_{\infty}} \cdot\|f\|_{\infty}=\varepsilon .
\end{aligned}
$$


Thus, by Lemma $5.4,\left\{A_{n}(u ; f)\right\}$ converges in $L_{p}$-norm for every $1 \leq p$ $<\infty$.

Remarks. 1 . The function $F$ on $X \otimes[u]$ defined by $F(x, v)=\widetilde{v}(x)$ ceases to be well defined if $\widetilde{v}$ is replaced by $v$. However, it would be possible to avoid the usage of lifting if the group $[u]$ were countable, which, in general, is not true.

2. By Theorem A.5 in the Appendix, the function $F$ is measurable on $X \otimes[u]$ only if $u$ assumes countably many values. That is why in Theorem 5.5 we assume that $u \in U_{\mathrm{c}}$.

Corollary 5.6. Given any $u \in U_{\mathrm{c}}$ and $f \in L_{p}$, the averages $A_{n}(u ; f)$ converge in $L_{p}$-norm for every $1 \leq p<\infty$.

Proof. Fix $\varepsilon>0$, and let $h \in L_{\infty}$ be such that $\|f-h\|_{p}<\varepsilon$. By Theorem 5.5 , the averages $A_{n}(u ; h)$ converge in $L_{p}$. We also have

$$
\left\|A_{n}(u ; f)-A_{n}(u ; h)\right\|_{p} \leq \frac{1}{n} \sum_{k=0}^{n-1}\left\|u^{k}\right\|_{\infty}\|f-h\|_{p}<\varepsilon .
$$

Therefore, by Lemma 5.4, we obtain $L_{p}$-convergence of $A_{n}(u ; f)$.

Corollary 5.7. For every function $u \in U_{\mathrm{c}}$, the averages

$$
A_{n}(u)=\frac{1}{n} \sum_{k=0}^{n-1} u^{k} \circ T^{k}
$$

converge in $L_{p}$-norm for every $1 \leq p<\infty$.

Assume now that $G$ is a subset of the group $U$.

Definition 5.8. $P_{s}: \mathbb{Z} \rightarrow L_{\infty}$ is called a trigonometric polynomial over $G$ if

$$
P_{s}(\cdot)=\sum_{j=1}^{s} r_{j} u_{j}^{(\cdot)}
$$

for some $\left\{r_{j}\right\}_{j=1}^{s} \subset \mathbb{C}$ and $\left\{u_{j}\right\}_{j=1}^{s} \subset G$.

By linearity, from Corollary 5.6 we obtain the following.

Proposition 5.9. Let $P_{s}$ be a trigonometric polynomial over the group $U_{\mathrm{c}}$. Then, for every $f \in L_{p}$, the averages

$$
A_{n}\left(P_{s} ; f\right)=\frac{1}{n} \sum_{k=0}^{n-1}\left(P_{s}(k) f\right) \circ T^{k}
$$

converge in $L_{p}$ for all $1 \leq p<\infty$.

Definition 5.10. Let $1 \leq p \leq \infty$. A sequence $\left\{b_{k}\right\} \subset L_{p}$ is called a $G_{p}$-Besicovitch $\left(G_{p}\right)$ sequence if for every $\varepsilon>0$ there is a trigonometric 
polynomial $P_{s}$ over $G$ such that

$$
\limsup _{n} \frac{1}{n} \sum_{k=0}^{n-1}\left\|b_{k}-P_{s}(k)\right\|_{p}<\varepsilon .
$$

A $G_{p}$ sequence $\left\{b_{k}\right\}$ is called bounded if there exists a constant $C$ such that $\left\|b_{k}\right\|_{\infty} \leq C<\infty$ for every $k$.

TheOREM 5.11. Let $1 \leq q \leq p<\infty$ be such that $1 / p+1 / q=1$. If $\left\{b_{k}\right\}$ is a $\left(U_{\mathrm{c}}\right)_{p}$ sequence and $f \in L_{\infty}$, then the averages

$$
A_{n}\left(\left\{b_{k}\right\} ; f\right)=\frac{1}{n} \sum_{k=0}^{n-1}\left(b_{k} f\right) \circ T^{k}
$$

converge in $L_{p}$-norm. For $f \in L_{p}$, the averages $A_{n}\left(\left\{b_{k}\right\} ; f\right)$ converge in $L_{p}$-norm if $\left\{b_{k}\right\}$ is a bounded $\left(U_{\mathrm{c}}\right)_{p}$ sequence. If $\left\{b_{k}\right\}$ is a $\left(U_{\mathrm{c}}\right)_{p}$ sequence with $\left\|b_{k}\right\|_{q} \leq C<\infty$ for every $k$, then these averages converge in $L_{1}$ for all $f \in L_{p}$.

Proof. Let $\left\{b_{k}\right\}$ be a $\left(U_{\mathrm{c}}\right)_{p}$ sequence and assume that $f \in L_{\infty}$. Fix $\varepsilon>0$ and choose $P_{s}$ such that $(* *)$ is satisfied. Then

$$
\begin{aligned}
\left\|A_{n}\left(\left\{b_{k}\right\} ; f\right)-A_{n}\left(P_{s} ; f\right)\right\|_{p} & \leq \frac{1}{n} \sum_{k=0}^{n-1}\left\|\left(b_{k}-P_{s}(k)\right) f\right\|_{p} \\
& \leq \frac{1}{n} \sum_{k=0}^{n-1}\left\|b_{k}-P_{s}(k)\right\|_{p}\|f\|_{\infty}<\varepsilon\|f\|_{\infty}
\end{aligned}
$$

for all $n$ large enough. Hence, by Lemma 5.4 and Proposition 5.9, the averages $A_{n}\left(\left\{b_{k}\right\} ; f\right)$ converge in $L_{p}$-norm.

Now, let $\left\{b_{k}\right\}$ be a bounded $\left(U_{\mathrm{c}}\right)_{p}$ sequence, and let $f \in L_{p}$. Fix $\varepsilon>0$ and pick $h \in L_{\infty}$ such that $\|f-h\|_{p}<\varepsilon$. By the first part of this proof, the sequence $\left\{A_{n}\left(\left\{b_{k}\right\} ; h\right)\right\}$ converges in $L_{p}$. Moreover,

$$
\left\|A_{n}\left(\left\{b_{k}\right\} ; f\right)-A_{n}\left(\left\{b_{k}\right\} ; h\right)\right\|_{p} \leq\left\|b_{k}\right\|_{\infty}\|f-h\|_{p}<C \varepsilon,
$$

which, by Lemma 5.4, yields the desired convergence.

Finally, let $\left\{b_{k}\right\}$ be a $\left(U_{\mathrm{c}}\right)_{p}$ sequence with $\left\|b_{k}\right\|_{q} \leq C<\infty$ for every $k$. Let $f \in L_{p}$. Given $\varepsilon>0$, one can find $h \in L_{\infty}$ with $\|f-h\|_{p}<\varepsilon$. Again, we know that $\left\{A_{n}\left(\left\{b_{k}\right\} ; h\right)\right\}$ converges in $L_{1}$. Also,

$$
\left\|A_{n}\left(\left\{b_{k}\right\} ; f\right)-A_{n}\left(\left\{b_{k}\right\} ; h\right)\right\|_{1} \leq\left\|b_{k}\right\|_{q}\|f-h\|_{p}<\varepsilon C,
$$

and the proof is complete by Lemma 5.4 .

6. Almost everywhere convergence along generalized Besicovitch weights. In this section we will study almost everywhere convergence of the averages of the type $(*)$. It turns out that the tools available are far 
from leading to a conclusive result if one considers the whole group $U$. Instead, we will restrict ourselves to the subgroup $U_{\mathrm{F}}$ of $U_{\mathrm{c}}$ consisting of all unimodular functions that assume a finite number of values.

Proposition 6.1. For every $u \in U_{\mathrm{F}}$ and any function $f \in L_{1}$, the averages $(*)$ converge almost everywhere.

Proof. Pick $u \in U_{\mathrm{F}}$, and let $\left\{\lambda_{1}, \ldots, \lambda_{m}\right\}$ with $\lambda_{i} \neq \lambda_{j}, i \neq j$, be the range of $u$. Then $u=\sum_{i=1}^{m} \lambda_{i} \chi_{E_{i}}$, where $E_{i}=u^{-1}\left(\lambda_{i}\right), i=1, \ldots, m$, which implies that

$$
u^{k}=\sum_{i=1}^{m} \lambda_{i}^{k} \chi_{E_{i}}
$$

for every $k$. Therefore,

$$
A_{n}(u ; f)(x)=\frac{1}{n} \sum_{k=0}^{n-1}\left(\sum_{i=1}^{m} \lambda_{i}^{k} \chi_{E_{i}} f\right)\left(T^{k} x\right)=\sum_{i=1}^{m} \frac{1}{n} \sum_{k=0}^{n-1} \lambda_{i}^{k} f_{i}\left(T^{k} x\right),
$$

where we set $f_{i}=f \chi_{E_{i}}$, so $f_{i} \in L_{1}$. Since the averages $n^{-1} \sum_{k=0}^{n-1} \lambda_{i}^{k} f_{i}\left(T^{k} x\right)$ converge a.e. $[\mathrm{Ry}]$ for every $i$, we get the desired convergence.

Remarks. 1. One can check that if $u \in U$, then the Dunford-Schwartz operator $T_{u}$ given by $\left(T_{u} f\right)(x)=(u f)(T x), f \in L_{1}$, does not generate the averages $(*)$, so one cannot employ the idea of the proof in [LO] to obtain the almost everywhere convergence of $(*)$ for $u \in U$.

2. It may seem that, due to Lemma 6.3 below, applying the argument of Theorem 5.5 one can strengthen the result of Proposition 6.1 if $G$ consists of elements $u$ such that $\left\{u^{k}: k \in \mathbb{Z}\right\}$ is precompact in $L_{\infty}$. But the $L_{\infty}$ precompactness of $\left\{u^{k}\right\}$ implies that $u \in U_{\mathrm{F}}$ :

Proposition 6.2. If $u \in U$, then the group $\left\{u^{k}: k \in \mathbb{Z}\right\}$ is precompact in $L_{\infty}$ if and only if $u$ assumes finitely many values.

Proof. The "if" part is obvious. Now, let $\left\{u^{k}: k \in \mathbb{Z}\right\}$ be precompact in $L_{\infty}$. Then, by the spectral theorem, $\left\{z^{k}: k \in \mathbb{Z}\right\}$ is precompact in $C(\Delta)$, where $\Delta$ is the closure of the range of $u$. Assume that $\Delta$ is not finite. Then one can find a sequence $\left\{\lambda_{n}\right\}_{n=1}^{\infty} \subset \Delta$ converging to some $\lambda_{0} \in \Delta$ such that $\lambda_{n} \neq \lambda_{0}$ for every $n$. By Arzelà's theorem, given $\varepsilon>0$, there exists $\delta>0$ such that $\left|z^{k}-w^{k}\right|<\varepsilon$ for all $k$ whenever $|z-w|<\delta$. Let $\varepsilon=1$; there exists $n_{0}$ such that for $n>n_{0}$ we have $\left|\lambda_{n}-\lambda_{0}\right|<\delta$, so $\left|\left(\lambda_{n} \lambda_{0}^{-1}\right)^{k}-1\right|<1$ for every $k$. But this is possible only if $\lambda_{n} \lambda_{0}^{-1}=1$ for $n>n_{0}$, a contradiction. Therefore, $\Delta$ is finite, and hence so is the range of $u$.

To proceed with the almost everywhere convergence along generalized Besicovitch weights we will need the following generalization of Lemma 5.4. Note that in [Ry] a version of this result is used implicitly. However, due to its important role, we found it worthwhile to state and prove it separately. 
LEMma 6.3. Let $\left\{A_{n}\right\}$ be a sequence of measurable functions such that, given $\varepsilon, \delta>0$, there exist a set $E \subset X$ with $\mu\left(E^{\mathrm{c}}\right)<\varepsilon$, an almost everywhere convergent sequence $\left\{\widehat{A}_{n}\right\}$ and a positive integer $N$ with

$$
\left\|\left(A_{n}-\widehat{A}_{n}\right) \chi_{E}\right\|_{\infty}<\delta \quad \text { for all } n \geq N .
$$

Then $\left\{A_{n}\right\}$ itself converges almost everywhere. In particular, $\left\{A_{n}\right\}$ converges a.e. if the above condition holds with $E=X$.

Proof. By Egorov's theorem, given $\varepsilon, \delta>0$, it is enough to find $E \subset X$ with $\mu\left(E^{\mathrm{c}}\right)<\varepsilon$ and a number $N$ such that

$$
\left\|\left(A_{m}-A_{n}\right) \chi_{E}\right\|_{\infty}<\delta \quad \text { whenever } m, n \geq N .
$$

By the assumption, we can find an almost everywhere convergent sequence $\left\{\widehat{A}_{n}\right\}$, a set $E_{1} \subset X$ with $\mu\left(E_{1}^{\mathrm{c}}\right)<\varepsilon / 2$, and a number $N_{1}$ such that

$$
\left\|\left(A_{n}-\widehat{A}_{n}\right) \chi_{E_{1}}\right\|_{\infty}<\delta / 3 \quad \text { when } n \geq N_{1} \text {. }
$$

Then, by Egorov's theorem, find $E_{2} \subset X$ with $\mu\left(E_{2}^{\mathrm{c}}\right)<\varepsilon / 2$ such that $\widehat{a}_{n}$ converges uniformly on $E_{2}$. Therefore, it is possible to find a number $N_{2}$ for which

$$
\left\|\left(\widehat{A}_{m}-\widehat{A}_{n}\right) \chi_{E_{2}}\right\|_{\infty}<\delta / 3 \quad \text { whenever } m, n \geq N_{2} .
$$

Finally, letting $N=\max \left\{N_{1}, N_{2}\right\}$ and $E=E_{1} \cap E_{2}$, we get $\mu\left(E^{\mathrm{c}}\right)<\varepsilon$ and

$$
\begin{aligned}
& \left\|\left(A_{m}-A_{n}\right) \chi_{E}\right\|_{\infty} \\
& \quad \leq\left\|\left(A_{m}-\widehat{A}_{m}\right) \chi_{E}\right\|_{\infty}+\left\|\left(\widehat{A}_{m}-\widehat{A}_{n}\right) \chi_{E}\right\|_{\infty}+\left\|\left(A_{n}-\widehat{A}_{n}\right) \chi_{E}\right\|_{\infty}<\delta
\end{aligned}
$$

as soon as $m, n \geq N$.

Now, by linearity, from Proposition 6.1 we obtain the following result.

Proposition 6.4. Let $P_{s}$ be a trigonometric polynomial over the group $U_{\mathrm{F}}$. Then the averages $A_{n}\left(P_{s} ; f\right)$ converge a.e. for every $f \in L_{1}$.

TheOREM 6.5. If $\left\{b_{k}\right\}$ is a $\left(U_{\mathrm{F}}\right)_{\infty}$ sequence (Definition 5.7), then the averages $A_{n}\left(\left\{b_{k}\right\} ; f\right)$ converge a.e. for every $f \in L_{\infty}$. If $\left\{b_{k}\right\}$ is a bounded $\left(U_{\mathrm{F}}\right)_{\infty}$ sequence, then these averages converge for all $f \in L_{1}$.

Proof. Fix $\varepsilon>0$, and let $P_{s}$ be such that condition (**) is satisfied. By Proposition 6.4 , the averages $A_{n}\left(P_{s} ; f\right)$ converge a.e. Further,

$$
\begin{aligned}
\left\|A_{n}\left(\left\{b_{k}\right\} ; f\right)-A_{n}\left(P_{s} ; f\right)\right\|_{\infty} & =\left\|\frac{1}{n} \sum_{k=0}^{n-1}\left(b_{k} f\right) \circ T^{k}-\frac{1}{n} \sum_{k=0}^{n-1}\left(P_{s}(k) f\right) \circ T^{k}\right\|_{\infty} \\
& \leq \frac{1}{n} \sum_{k=0}^{n-1}\left\|\left(\left(b_{k}-P_{s}(k)\right) f\right) \circ T^{k}\right\|_{\infty} \\
& \leq \frac{1}{n} \sum_{k=0}^{n-1}\left\|b_{k}-P_{s}(k)\right\|_{\infty}\|f\|_{\infty} .
\end{aligned}
$$


Therefore, for large enough $n$, we obtain

$$
\begin{aligned}
\left\|A_{n}\left(\left\{b_{k}\right\} ; f\right)-A_{n}\left(P_{s} ; f\right)\right\|_{\infty} & \leq \limsup _{n} \frac{1}{n} \sum_{k=0}^{n-1}\left\|b_{k}-P_{s}(k)\right\|_{\infty} \cdot\|f\|_{\infty} \\
& <\varepsilon\|f\|_{\infty},
\end{aligned}
$$

which, by Lemma 6.3 , completes the first part of the proof.

Now, observe that, if $\left\{b_{k}\right\}$ is a bounded $\left(U_{\mathrm{F}}\right)_{\infty}$ sequence, then, for every $f \in L_{1}$,

$$
\left|A_{n}\left(\left\{b_{k}\right\} ; f\right)(x)\right|=\left|\frac{1}{n} \sum_{k=0}^{n-1} b_{k}\left(T^{k} x\right) \cdot f\left(T^{k} x\right)\right| \leq\left\|b_{k}\right\|_{\infty} \cdot \frac{1}{n} \sum_{k=0}^{n}|f|\left(T^{k} x\right)
$$

almost everywhere, and the averages $n^{-1} \sum_{k=0}^{n-1}|f|\left(T^{k} x\right)$ converge almost everywhere by the pointwise ergodic theorem for $|f| \in L_{1}$. Hence, $\sup _{n}\left|A_{n}\left(\left\{b_{k}\right\} ; f\right)(x)\right|<\infty$ a.e. on $X$, for all $f \in L_{1}$. Since we have the a.e. convergence of $A_{n}\left(\left\{b_{k}\right\} ; f\right)$ for all $f \in L_{\infty}$, and $L_{\infty}$ is dense in $L_{1}$, by the Banach principle, the assertion follows.

REMARK. If one replaces $U_{\mathrm{F}}$ with $\{u \in U: u(x) \equiv \lambda,|\lambda|=1\}$, then the classical result $[\mathrm{Ry}]$ follows: if $\left\{\beta_{k}\right\} \subset \mathbb{C}$ is a bounded Besicovitch sequence, then the weighted averages

$$
A_{n}\left(\left\{\beta_{k}\right\} ; f\right)(x)=\frac{1}{n} \sum_{k=0}^{n-1} \beta_{k} f\left(T^{k} x\right)
$$

converge almost everywhere on $X$ for all $f \in L_{1}$.

Now, using the tools employed to obtain the basic result in Section 2, we generalize Theorem 6.5 to the setting of $T$-admissible processes.

Theorem 6.6. Let $F=\left\{f_{n}\right\} \subset L_{1}$ be a bounded T-admissible process, where $T$ is an invertible MPT, and $\left\{b_{i}\right\}$ be a bounded $\left(U_{\mathrm{F}}\right)_{\infty}$ sequence. Then the averages

$$
A_{n}\left(\left\{b_{i}\right\} ; F\right)(x):=\frac{1}{n} \sum_{i=0}^{n-1} b_{i}\left(T^{i} x\right) f_{i}(x)
$$

converge almost everywhere on $X$.

Proof. We use the same machinery as in Theorem 2.2. For a fixed $m \in \mathbb{Z}^{+}$, define

$$
g_{i}^{m}(x)= \begin{cases}f_{m}\left(T^{i-m} x\right) & \text { for } i>m, \\ f_{i}(x) & \text { for } 0 \leq i \leq m .\end{cases}
$$


Hence $f_{i}-g_{i}^{m} \leq T^{i} c_{m}$ and

$$
D_{n}:=\frac{1}{n} \sum_{i=0}^{n-1}\left(f_{i}-g_{i}^{m}\right) \leq \frac{1}{n} \sum_{i=0}^{n-1} T^{i} c_{m}, \quad \text { where } \quad c_{m}=\delta-v_{m} .
$$

Now, let

$$
f^{*}(x)=\limsup _{n} A_{n}\left(\left\{b_{i}\right\} ; F\right)(x), \quad f_{*}(x)=\liminf _{n} A_{n}\left(\left\{b_{i}\right\} ; F\right)(x) .
$$

Then

$$
\begin{aligned}
0 \leq\left|f^{*}(x)-f_{*}(x)\right| & \leq \underset{n}{2 \limsup }\left|A_{n}\left(\left\{b_{i}\right\} ; F\right)(x)-A_{n}\left(\left\{b_{i}\right\} ;\left\{g_{i}^{m}\right\}\right)(x)\right| \\
& \leq 2 \underset{n}{\limsup }\left|\frac{1}{n} \sum_{i=0}^{n-1} b_{i}\left(T^{i} x\right)\left(f_{i}-g_{i}^{m}\right)(x)\right| \\
& \leq 2 B \limsup _{n} D_{n}(x) .
\end{aligned}
$$

Given $\alpha>0$, define $E=\left\{\omega: f^{*}(x)-f_{*}(x)>\alpha\right\}$. In order to prove a.e. convergence of $A_{n}\left(\left\{b_{i}\right\} ; F\right)$ it is enough to show that $\mu(E)=0$. First, $\lim _{n} A_{n}\left(\left\{b_{i}\right\} ; g_{i}^{m}\right)(x)$ exists a.e. by Theorem 6.5. Thus,

$$
E \subset\left\{x: \sup _{n}\left|D_{n}(x)\right|>\frac{\alpha}{2 B}\right\} \subset\left\{x: \sup _{n}\left|\frac{1}{n} \sum_{i=0}^{n-1} T^{i} c_{m}(x)\right|>\frac{\alpha}{2 B}\right\} .
$$

By the a.e. convergence, the additive process $\left\{\sum_{i=0}^{n-1} T^{i} c_{m}\right\}$ admits a maximal inequality along $\left\{b_{i}\right\}$. Therefore, for some constant $C$,

$$
\mu(E) \leq \mu\left(\left\{x: \sup _{n}\left|\frac{1}{n} \sum_{i=0}^{n-1} T^{i} c_{m}(x)\right|>\frac{\alpha}{2 B}\right\}\right) \leq \frac{2 B C}{\alpha}\left\|c_{m}\right\|_{1} .
$$

Since $\left\|c_{m}\right\|_{1} \downarrow 0$ as $m \rightarrow \infty$, we obtain $\mu(E)=0$.

As mentioned earlier, the Wiener-Wintner theorem implies that, for any function $u \in U$, the averages $n^{-1} \sum_{k=0}^{n-1} u^{k}(x) f\left(T^{k} x\right)$ converge a.e. for all $f \in L_{1}$. Consequently, for all $f \in L_{1}$ and any trigonometric polynomial $P_{s}$ over $U$, the averages

$$
\frac{1}{n} \sum_{k=0}^{n-1} P_{s}(k)(x) f\left(T^{k} x\right)
$$

converge a.e. Therefore, one can also define weights using linear combinations of $u^{k}$ 's, $u \in U$, and ask whether such weights are good for a.e. convergence. The following theorems are obtained by using the methods used above in this section verbatim. Hence, we will state them only.

TheOREm 6.7. Let $\left\{b_{k}\right\}$ be a bounded $U_{\infty}$ sequence (Definition 5.10). For every $f \in L_{1}$ the weighted averages $n^{-1} \sum_{k=0}^{n-1} b_{k}(x) f\left(T^{k} x\right)$ converge almost everywhere on $X$. 
TheOREM 6.8. Let $F=\left\{f_{n}\right\} \subset L_{1}$ be a bounded T-admissible process, where $T$ is invertible, and $\left\{b_{k}\right\}$ be a bounded $U_{\infty}$ sequence. Then the averages $n^{-1} \sum_{k=0}^{n-1} b_{k}(x) f_{k}(x)$ converge almost everywhere on $X$.

7. Identification of the limits in Theorems 5.11, 6.5, and 6.7. As in $[\mathrm{Ry}]$, we will identify the limits when $T$ is a weakly mixing transformation. Recall that a measure preserving transformation $T$ is weakly mixing if and only if

(i) $T$ is ergodic, i.e. $f \in L_{1}(X), f \circ T=f$ imply $f=$ const; and

(ii) $f \in L_{1}(X), f \circ T=\lambda \cdot f, \lambda \neq 1$ imply $f=0$.

Proposition 7.1. Let $\left\{b_{k}\right\}$ be a bounded $\left(U_{\mathrm{c}}\right)_{p}$ sequence (Definition 5.10). Then the averages $A_{n}\left(\left\{b_{k}\right\} ; f\right)$ converge in $L_{p}$ to constants for every $f \in L_{p}$ and every $\left\{b_{k}\right\}$ if and only if the transformation $T$ is weakly mixing.

Proof. Repeating a standard argument [Ry], one can check that if the averages $A_{n}\left(\left\{b_{k}\right\} ; f\right)$ converge in $L_{p}$ to constants for all $f \in L_{p}$, then $T$ is weakly mixing.

Next, assume that $T$ is weakly mixing. Pick $u \in U_{\mathrm{c}}$, and let $f \in L_{p}$. By Corollary 5.6, we have

$$
A_{n}(u ; f)=\frac{1}{n} \sum_{k=0}^{n-1}\left(u^{k} f\right) \circ T^{k} \rightarrow f_{u} \quad \text { in } L_{p} .
$$

We shall show that $f_{u}$ is constant. Applying the spectral theorem to the unitary function $u$, one has

$$
u=\|\cdot\|_{\infty^{-}} \lim _{m \rightarrow \infty} \sum_{j=1}^{m} \lambda_{j}(m) \chi_{E_{j}(m)}
$$

where $\left|\lambda_{j}(m)\right|=1$ for all $m$ and $j$, and $\mu\left(E_{j}(m) \cap E_{j^{\prime}}(m)\right)=0$ for every $m$ whenever $j \neq j^{\prime}$. Therefore, for a fixed $k$,

$$
\sum_{j=1}^{m} \lambda_{j}^{k}(m) \chi_{E_{j}(m)}=\left[\sum_{j=1}^{m} \lambda_{j}(m) \chi_{E_{j}(m)}\right]^{k} \rightarrow u^{k} \quad \text { in } L_{\infty}
$$

as $m \rightarrow \infty$, which implies that

$$
\left(\sum_{j=1}^{m} \lambda_{j}^{k}(m) \chi_{E_{j}(m)} f\right) \circ T^{k} \rightarrow\left(u^{k} f\right) \circ T^{k} \quad \text { in } L_{p}
$$

for every $k$ as $m \rightarrow \infty$. It now follows that

$$
f_{m n}=\frac{1}{n} \sum_{k=0}^{n-1}\left(\sum_{j=1}^{m} \lambda_{j}^{k}(m) \chi_{E_{j}(m)} f\right) \circ T^{k} \rightarrow a_{n}(u ; f) \quad \text { in } L_{p}
$$


as $m \rightarrow \infty$. On the other hand,

$$
f_{m n}=\sum_{j=1}^{m} \frac{1}{n} \sum_{k=0}^{n-1} \lambda_{j}^{k}(m) f_{j}(m) \circ T^{k}
$$

where $f_{j}(m)=\chi_{E_{j}(m)} f\left(\in L_{p}\right)$. By Theorem 7 in $[\mathrm{Ry}]$, the averages $n^{-1} \sum_{k=0}^{n-1} \lambda_{j}^{k}(m) f_{j}(m) \circ T^{k}$ converge a.e. to a constant for all $j$. Therefore, by the bounded convergence theorem, it can be easily seen that these averages converge to a constant in $L_{p}$ for every $j$. Thus,

$$
f_{m n} \rightarrow c_{m}=\text { const }, \quad n \rightarrow \infty .
$$

Now, due to (1)-(3), it is possible to find a subsequence $c_{m_{l}}$ converging to $f_{u}$ in $L_{p}$. Then it can be easily verified that $f_{u}$ is constant.

Let now $\left\{b_{k}\right\}$ be a bounded $\left(U_{\mathrm{c}}\right)_{p}$ sequence. Then for every $m$ one can find a trigonometric polynomial $P_{s(m)}$ over $U$ such that

$$
\limsup _{n} \frac{1}{n} \sum_{k=0}^{n-1}\left\|b_{k}-P_{s(m)}(k)\right\|_{\infty}<\frac{1}{m} .
$$

For $h \in L_{\infty}$, defining

$$
A_{m n}=A_{n}\left(P_{s(m)} ; h\right)=\frac{1}{n} \sum_{k=0}^{n-1}\left(P_{s(m)}(k) h\right) \circ T^{k},
$$

we get

$$
\left\|A_{n}\left(\left\{b_{k}\right\} ; h\right)-A_{m n}\right\|_{p} \leq \frac{1}{n} \sum_{k=0}^{n-1}\left\|b_{k}-P_{s(m)}(k)\right\|_{p} \cdot\|h\|_{\infty}<\frac{\|h\|_{\infty}}{m}
$$

for all $n \geq n(m)$. Therefore, for some subsequence $\left\{n_{m}\right\}$, we have

$$
\left\|A_{n_{m}}\left(\left\{b_{k}\right\} ; h\right)-A_{m n_{m}}\right\|_{p} \rightarrow 0, \quad m \rightarrow \infty .
$$

At the same time, by the first part of the proof,

$$
A_{m n_{m}} \rightarrow A_{m}=\mathrm{const} \quad \text { in } L_{p}
$$

as $m \rightarrow \infty$. Since we also know that the sequence $\left\{A_{n_{m}}\left(\left\{b_{k}\right\} ; h\right)\right\}$ converges in $L_{p}$ to some $\bar{h}$, taking (4) and (5) into account, we infer that $\bar{h}$ is constant.

Finally, let $\left\{h_{m}\right\} \subset L_{\infty}$ be such that $\left\|f-h_{m}\right\|_{p}<1 / m$. Then, for every $m$ and $n$, we have

$$
\left\|A_{n}\left(\left\{b_{k}\right\} ; f\right)-A_{n}\left(\left\{b_{k}\right\} ; h_{m}\right)\right\|_{p} \leq\left\|b_{k}\right\|_{\infty} \cdot\left\|f-h_{m}\right\|_{p}<C / m .
$$

Remembering that $\left\{A_{n}\left(\left\{b_{k}\right\} ; f\right)\right\}$ converges in $L_{p}$, one can see now that it must converge to a constant.

TheOREM 7.2. Let $\left\{b_{k}\right\}$ be a bounded $\left(U_{\mathrm{F}}\right)_{\infty}$ sequence. Then the averages $A_{n}\left(\left\{b_{k}\right\} ; f\right)$ converge a.e. to constants for every $f \in L_{1}$ if and only if $T$ is weakly mixing. 
Proof. Again, it is enough to show the "if" part. If $T$ is weakly mixing, since $U_{\mathrm{F}} \subset U_{\mathrm{c}}$, by Theorem 7.1, the averages $A_{n}\left(\left\{b_{k}\right\} ; f\right)$ converge to constants in $L_{1}$ for all $f \in L_{1}$. Since we also know that these averages converge a.e. to some $\bar{f} \in L_{1}$, it follows that $\bar{f}$ is constant.

In a similar fashion we obtain the following result.

THEOREM 7.3. If $\left\{b_{k}\right\}$ is a bounded $\left(U_{\mathrm{F}}\right)_{\infty}$ sequence, then the averages $n^{-1} \sum_{k=0}^{n-1} b_{k}(x) f\left(T^{k} x\right)$ converge a.e. to constants for every $f \in L_{1}$ if and only if $T$ is weakly mixing.

Appendix: A measurability criterion. The results of this section are due to A. Ber and V. Chilin (see Acknowledgements).

Let $G$ be a compact group equipped with the Borel $\sigma$-algebra. If $\widehat{G}$ is the group of all continuous characters on $G$, then the following two results are known.

Theorem A.1 (see [HR, Corollary 22.19]). For a measurable character $\phi: G \rightarrow \mathbb{C}$, we have $\phi \in \widehat{G}$.

Theorem A.2 (see [HR, Theorem 24.15]). If $G$ is Abelian, then it is metrizable if and only if $\widehat{G}$ is countable.

Let $G$ be a metrizable compact subgroup of $U=\left\{v \in L_{\infty}(X):|v(x)|=1\right.$ a.e. $\}$. Let $\mathcal{B}$ be the Borel $\sigma$-algebra on $G$. Define a function $F: X \otimes G \rightarrow \mathbb{C}$ by $F(x, v)=\widetilde{v}(x)$ (here $L_{\infty} \ni g \mapsto \widetilde{g} \in \mathbb{L}_{\infty}$ is a lifting; see Section 5). Also, if $x \in X$, we define $\widehat{x}: G \rightarrow \mathbb{C}$ by $\widehat{x}(v)=\widetilde{v}(x)$. Note that $\widehat{x}$ is a character on $G$ for every $x \in X$.

Theorem A.3. If $\widehat{x}$ is measurable on $G$ for every $x \in X$, then there exists a d.m.p. $\left\{X_{n}\right\}_{n=1}^{\infty}$ of $X$ (see Section 5) such that, for any $v \in G$ and $n$, there is a constant $c_{n}(v)$ such that $v(x)=c_{n}(v)$ a.e. on $X_{n}$. Moreover, the function $F(x, v)=\widetilde{v}(x)$ is measurable on $X \otimes G$.

Proof. By Theorem A.1, $\widehat{x} \in \widehat{G}$ for every $x \in X$. Furthermore, since $G$ is Abelian and metrizable, Theorem A.2 shows that $\widehat{G}=\left\{\phi_{n}\right\}_{n=1}^{\infty}$, where $\phi_{i} \neq \phi_{j}$ whenever $i \neq j$. Set $\widehat{x}=\Phi(x)$, and let

$$
A_{n}=\Phi^{-1}\left(\phi_{n}\right), \quad n=1,2, \ldots
$$

Then $A_{i} \cap A_{j}=\emptyset$ if $i \neq j$ and $X=\bigcup A_{n}$. Pick $x_{n} \in A_{n}, n=1,2, \ldots$, arbitrarily. Since

$$
\begin{aligned}
x \in A_{n} & \Leftrightarrow \Phi(x)=\phi_{n}\left(=\Phi\left(x_{n}\right)\right) \Leftrightarrow \widehat{x}=\widehat{x}_{n} \\
& \Leftrightarrow \widetilde{v}(x)=\widetilde{v}\left(x_{n}\right) \forall v \in G \Leftrightarrow x \in(\widetilde{v})^{-1}\left(\widetilde{v}\left(x_{n}\right)\right) \forall v \in G,
\end{aligned}
$$

we have

$$
A_{n}=\bigcap_{v \in G}(\widetilde{v})^{-1}\left(\widetilde{v}\left(x_{n}\right)\right) .
$$


Also, since $G$ is metrizable and compact, it is separable. Let $G=\overline{\left\{v_{m}\right\}_{m=1}^{\infty}}$. If we define

$$
X_{n}=\bigcap_{m=1}^{\infty}\left(\widetilde{v}_{m}\right)^{-1}\left(\widetilde{v}_{m}\left(x_{n}\right)\right), \quad n=1,2, \ldots,
$$

then each $X_{n}$ is measurable.

We now show that $X_{n}=A_{n}$ for every $n$. Indeed, because $A_{n} \subset X_{n}$ and $X=\bigcup A_{n}$, it is enough to prove that $X_{i} \cap X_{j}=\emptyset$ if $i \neq j$. Assume that $x \in X_{i} \cap X_{j}$ and $i \neq j$. Then $\widetilde{v}_{m}(x)=\widetilde{v}_{m}\left(x_{i}\right)$ and, at the same time, $\widetilde{v}_{m}(x)=\widetilde{v}_{m}\left(x_{j}\right)$, that is, $\widetilde{v}_{m}\left(x_{i}\right)=\widetilde{v}_{m}\left(x_{j}\right)$, or $\widehat{x}_{i}\left(v_{m}\right)=\widehat{x}_{j}\left(v_{m}\right)$ for all $m$. Since $\left\{v_{m}\right\}$ is dense in $G$ and $\widehat{x}_{i}$ and $\widehat{x}_{j}$ are continuous on $G$, we get $\widehat{x}_{i}=\widehat{x}_{j}$, which implies that $A_{i}=A_{j}$, a contradiction. Therefore, $\left\{X_{n}\right\}$ is a d.m.p. of $X$ (we discard the sets $X_{i}$ with zero measure). If we define $\widetilde{v}\left(x_{n}\right)=c_{n}(v)$, then for any $x \in X_{n}$ and $v \in G$ we have $\widetilde{v}(x)=\widetilde{v}\left(x_{n}\right)=c_{n}(v)$, so $v(x)=$ $c_{n}(v)$ a.e. on $X_{n}$.

We now show that the function $F$ is measurable. Let $B$ be any Borel set in $\mathbb{C}$. Since $x \in X_{n}$ is equivalent to $\widetilde{v}(x)=\widetilde{v}\left(x_{n}\right)$ for all $v \in G$, we have

$$
\begin{aligned}
F^{-1}(B) & =F^{-1}(B) \cap \bigcup_{n=1}^{\infty}\left(X_{n} \times G\right)=\bigcup_{n=1}^{\infty}\left(F^{-1}(B) \cap\left(X_{n} \times G\right)\right) \\
& =\bigcup_{n=1}^{\infty}\left\{(x, v) \in X_{n} \times G: \widetilde{v}(x) \in B\right\} \\
& =\bigcup_{n=1}^{\infty}\left(X_{n} \times\left\{v \in G: \widetilde{v}\left(x_{n}\right) \in B\right\}\right) \in \Sigma \otimes \mathcal{B},
\end{aligned}
$$

because, by our assumption, the set $\left\{v \in G: \widetilde{v}\left(x_{n}\right) \in B\right\}=\{v \in G$ : $\left.\widehat{x}_{n}(v) \in B\right\}$ is measurable.

THEOREM A.4. Let the metric in $G$ be generated by the $L_{1}$-norm. If there exists a d.m.p. $\left\{X_{n}\right\}$ of $X$ such that, for every $v \in G$ and $n$, there is a constant $c_{n}(v)$ such that $v(x)=c_{n}(v)$ a.e. on $X_{n}$, then the function $F(x, v)=\widetilde{v}(x)$ is measurable on $X \otimes G$.

Proof. We will write $\chi_{E}$ for the characteristic function of a set $E \in \Sigma$, while $\left[\chi_{E}\right]$ will denote its class in $L_{\infty}(X)$. Obviously, $\left[\chi_{X_{n}}\right] \sim=\chi_{E_{n}}$ for some $E_{n} \in \Sigma$. Also $\mu\left(X_{n} \triangle E_{n}\right)=0$ and $\left[\chi_{E_{n}}\right]^{\sim}=\chi_{E_{n}}$, for $n=1,2, \ldots$ Define $Y_{1}=E_{1}$ and $Y_{n}=E_{n} \backslash \bigcup_{i=i}^{n-1} E_{i}, n \geq 2$. Because $\chi_{Y_{n}}=\prod_{i=1}^{n-1} \chi_{E_{n}}\left(1-\chi_{E_{i}}\right)$, and lifting is a homomorphism, it follows that $\left[\chi_{Y_{n}}\right]^{\sim}=\chi_{Y_{n}}$ for every $n$. Since also $\mu\left(\bigcup_{n=1}^{\infty} Y_{n}\right)=1, Y_{i} \cap Y_{j}=\emptyset$ if $i \neq j$, and $v(x)=c_{n}(v)$ a.e. on $Y_{n}$ for every $n$, without loss of generality we can assume that $\left[\chi_{X_{n}}\right] \sim=\chi_{X_{n}}$, $n=1,2, \ldots$ Then $v\left[\chi_{X_{n}}\right]=c_{n}(v)\left[\chi_{X_{n}}\right]$ implies that $\widetilde{v}(x)=c_{n}(v)$ for all $x \in X_{n}$ and $v \in G$. 
By Theorem A.3, the proof will be finished if we show that the character $\widehat{x}$ is continuous on $G$ for every $x \in X$. Let $v_{k}, v \in G$ and $\left\|v_{k}-v\right\|_{1} \rightarrow 0$ as $k \rightarrow \infty$. For each pair $k$ and $m$ we have

$$
\left|v-v_{k}\right|\left[\chi_{X_{m}}\right]=\left|\left(v-v_{k}\right)\left[\chi_{X_{m}}\right]\right|=\left|c_{m}(v)-c_{m}\left(v_{k}\right)\right|,
$$

which implies that

$$
\int_{X_{m}}\left|v-v_{k}\right| d \mu=\left|c_{m}(v)-c_{m}\left(v_{k}\right)\right| \cdot \mu\left(X_{m}\right) .
$$

If $x \in X$, then there exists an index $n_{0}$ such that $x \in X_{n_{0}}$. Therefore,

$$
\begin{aligned}
\left|\widehat{x}(v)-\widehat{x}\left(v_{k}\right)\right| & \cdot \mu\left(X_{n_{0}}\right) \\
& =\left|\widetilde{v}(x)-\widetilde{v}_{k}(x)\right| \cdot \mu\left(X_{n_{0}}\right)=\left|c_{n_{0}}(v)-c_{n_{0}}\left(v_{k}\right)\right| \cdot \mu\left(X_{n_{0}}\right) \\
& =\int_{X_{n_{0}}}\left|v-v_{k}\right| d \mu \leq \int_{X}\left|v-v_{k}\right| d \mu=\left\|v-v_{k}\right\|_{1} \rightarrow 0,
\end{aligned}
$$

that is, $\widehat{x}$ is continuous on $G$ for every $x \in X$.

The following is a direct consequence of Theorems A.3 and A.4.

Theorem A.5. Let $(X, \Sigma, \mu)$ be a probability space, and let $G$ be an $L_{1}$-compact subgroup of the group $U$ of all unimodular measurable functions on $X$. Consider the Borel $\sigma$-algebra on $G$. Let $f \mapsto \widetilde{f}$ be a lifting on $L_{\infty}(X)$. Then the function $F: X \otimes G \rightarrow \mathbb{C}$ given by $F(x, v)=\widetilde{v}(x)$ is measurable if and only if there exists a d.m.p. $\left\{X_{n}\right\}$ of $X$ such that for every $v \in G$ and $n$ there is a constant $c_{n}(v)$ with $v(x)=c_{n}(v)$ a.e. on $X_{n}$.

Acknowledgements. 1. The authors would like to express their deep gratitude to the anonymous referee for his/her careful reading of the earlier version of the manuscript, several insightful comments and for the example in Section 5.

2. We are also thankful to Prof. V. Chilin and Dr. A. Ber of the National University of Uzbekistan, Tashkent, Uzbekistan, for kindly letting us include their results in the Appendix.

3. Part of this research was carried out during the first author's visits to Ben Gurion University of the Negev, Beer Sheva, Israel, and to Penn State University at Hazleton, Hazleton, PA. He would like to thank both institutions for their hospitality.

\section{References}

[AS] M. A. Akcoglu and L. Sucheston, A ratio ergodic theorem for superadditive processes, Z. Wahrsch. Verw. Gebiete 44 (1978), 269-278.

[BO] J. R. Baxter and J. Olsen, Weighted and subsequential ergodic theorems, Canad. J. Math. 35 (1983), 145-166. 
[BeL] A. Bellow and V. Losert, The weighted pointwise ergodic theorem and the individual ergodic theorem along subsequences, Trans. Amer. Math. Soc. 288 (1985), 307-345.

[Bo] J. Bourgain, Pointwise ergodic theorems for arithmetic sets, with an appendix on return times sequences jointly with H. Fürstenberg, Y. Katznelson and D. Ornstein, Inst. Hautes Etudes Sci. Publ. Math. 69 (1989), 5-45.

[BrK] A. Brunel and M. Keane, Ergodic theorems for operator sequences, Z. Wahrsch. Verw. Gebiete 12 (1969), 231-240.

[C] D. Çömez, General and weighted averages of admissible superadditive processes, Illinois J. Math. 43 (1999), 582-591.

[ÇF] D. Çömez and S. Ferrando, On the sequences that are good in the mean for positive $L_{p}$-contractions, $1 \leq p<\infty$, ibid. 42 (1998), 617-629.

[ÇLO] D. Çömez, M. Lin and J. Olsen, Weighted ergodic theorems for mean ergodic $L_{1}$-contractions, Trans. Amer. Math. Soc. 350 (1998), 101-117.

[HR] E. Hewitt and K. A. Ross, Abstract Harmonic Analysis I, Springer, Berlin, 1963.

[JO] R. Jones and J. Olsen, Multiparameter weighted ergodic theorems, Canad. J. Math. 46 (1994), 343-356.

[Ka] J. P. Kahane, Sur les coefficients de Fourier-Bohr, Studia Math. 21 (1961), 103106.

[Ku] A. G. Kusraev, Dominated Operators, Kluwer, Dordrecht, 2000.

[LO] M. Lin and J. Olsen, Besicovitch functions and weighted ergodic theorems for LCA group actions, in: Convergence in Ergodic Theory and Probability, V. Bergelson et al. (eds.), de Gruyter, Berlin, 1996, 277-289.

[LOT] M. Lin, J. Olsen and A. Tempelman, On modulated ergodic theorems for DunfordSchwartz operators, Illinois J. Math. 43 (1999), 542-567.

[Li] S. Litvinov, On weighted ergodic theorems in von Neumann algebras, Doctoral Dissertation, North Dakota State Univ., Fargo, 1999.

[Ru] D. Rudolph, A joinings proof of Bourgain's return time theorem, Ergodic Theory Dynam. Systems 14 (1994), 197-203.

[Ry] C. Ryll-Nardzewski, Topics in ergodic theory, in: Winter School on Probability (Karpacz, 1975), Lecture Notes in Math. 472, Springer, 1975, 131-157.

[T] A. Tempelman, Ergodic theorems for amplitude modulated homogeneous random fields, Lithuanian Math. J. 14 (1974), 221-229 (in Russian); English transl.: Lithuanian Math. Transl. 14 (1975), 698-704.

Department of Mathematics

North Dakota State University

Fargo, ND 58105-5075, U.S.A.

E-mail: dogan.comez@ndsu.nodak.edu
Department of Mathematics Penn State University at Hazleton Hazleton, PA 18202, U.S.A. E-mail: snl2@psu.edu

Received December 23, 2002

Revised version October 11, 2004 